\title{
Ronsard, la Fortune et la Providence: deux élégies au sujet de Mary Stuart, reine d'Écosse
}

Ian R. Morrison

\section{(2) OpenEdition}

1 Journals

\section{Édition électronique}

URL : https://journals.openedition.org/studifrancesi/25883

DOI : 10.4000/studifrancesi.25883

ISSN : 2421-5856

Éditeur

Rosenberg \& Sellier

\section{Édition imprimée}

Date de publication : 1 avril 2007

Pagination : 99-106

ISSN : 0039-2944

\section{Référence électronique}

Ian R. Morrison, «Ronsard, la Fortune et la Providence: deux élégies au sujet de Mary Stuart, reine

d'Écosse », Studi Francesi [En ligne], 151 (LI | I) | 2007, mis en ligne le 30 novembre 2015, consulté le 22 novembre 2021. URL : http://journals.openedition.org/studifrancesi/25883 ; DOI : https://doi.org/ 10.4000/studifrancesi.25883

\section{(c) (i) $\odot$}

Studi Francesi è distribuita con Licenza Creative Commons Attribuzione - Non commerciale - Non opere derivate 4.0 Internazionale. 


\section{Ronsard, la Fortune et la Providence: deux élégies au sujet de Mary Stuart, reine d'Écosse}

Issue d'une tradition antique et médiévale, la Fortune demeure vivace chez les auteurs français du XVI siècle. Mais la lointaine ascendance païenne de ce personnage peut inquiéter, à une époque où les théologiens sont bien méfiants; on sait, par exemple, qu'à Rome Montaigne dut justifier certains aspects des Essais, dont «l'esser ricorso alla parola 'fortuna'»'. En général, l'un des points les plus délicats est celui des rapports de la Fortune et de la Providence. Comme nous le verrons, deux élégies de Ronsard posent ce problème avec une acuité particulière. Avant d'aborder ces poèmes, toutefois, je me permets de rappeler brièvement l'essentiel de la question.

La Fortune (avec ou sans majuscule) est souvent une personnification du hasard, et surtout des vicissitudes les plus spectaculaires que l'on puisse attribuer à celui-ci. C'est ce lieu commun que rappelle par exemple Marot, quand il déclare à Lyon Jamet:

Je ne t'escry de Fortune puissante,

Tu voys assez s'elle est ferme ou glissante

(Épître X, v. 5-6)².

Comment la Fortune, ainsi conçue, se situe-t-elle par rapport à la Providence? Écoutons d'abord l'un des grands théologiens du siècle, Jean Calvin. Selon lui, Dieu dirige tout et la Fortune n'a pas d'existence objective: 'si toute prospérité est bénédiction de Dieu, adversité sa malédiction, il ne reste plus nul lieu à fortune en tout ce qui advient aux hommes' (Institution de la Religion Chrestienne, t. I, xvi, 8, p. 233) Cela dit, Calvin fait pourtant une distinction capitale:

Ie di donques, combien que toutes choses soyent conduites par le conseil de Dieu, toutesfois qu'elles nous sont fortuites. Non pas que nous réputions fortune dominer sur les hommes pour tourner haut et bas toutes choses témérairement (car ceste resverie doit estre loin d'un cœur Chrestien); mais pource que des choses qui adviennent, l'ordre, la raison, la fin et nécessité est le plus souvent cachée au conseil de Dieu et ne peut estre comprinse par l'opinion humaine (I, xvi, 9, p. 234).

(1) Viaggio in Italia, éd. G. Greco et E. Camesasca, Milano: RCSLibri, 2003, p. 267. Cf. Journal de voyage en Italie, éd. P. Michel, Paris, Livre de Poche, 1974, p. 288. Voir Y. Bellenger, La fortune dans les Essais de Montaigne, in Il tema della Fortuna nella letteratura francese e italiana del Rinascimento, éd. E. Balmas, Florence, Olschki, 1990, pp. 491-505.

(2) Epîtres, éd. C.A. Mayer, Londres, Athlone Press, 1958, p. 128.

(3) Institution de la Religion Chrestienne, éd. J.-D. Benoît, Paris, Vrin, 1957-63 5 tomes. 
Calvin admet donc que l'on puisse parler du 'fortuit' et de la fortune, pourvu qu'il ne s'agisse que de reconnaître par là la faiblesse de nos lumières humaines. Si, par exemple, un marchand s'égare dans une forêt et se fait tuer ainsi par des brigands, le chrétien, selon Calvin, 'réputera que cela est fortuit en sa nature, mais il ne doutera pas que la providence de Dieu n'ait présidé à guider la fortune à son but' (ibid.).

En accueillant ainsi la notion de fortune comme hasard apparent, Calvin est héritier en droite ligne de théologiens tels saint Augustin et saint Thomas d'Aquin ${ }^{4}$. Mais cette sorte d'aval théologique est loin de s'appliquer à tous les cas que l'on rencontre dans les textes littéraires. Dans la pratique, les auteurs se plaignent volontiers de la Fortune. Sur le plan théologique, de telles plaintes sont suspectes: si, en effet, on considère la Fortune comme un simple aspect de la Providence, tout auteur qui s'en prend à celle-là a bien l'air de s'en prendre également à celle-ci. Le dossier devient nettement plus délicat encore si un auteur se met à voir dans la Fortune une puissance plus ou moins autonome, qui échappe plus ou moins à la direction de la Providence, pour intervenir à sa guise dans la vie des hommes. Telle est, par exemple, la Fortune dont se plaint Brantôme: vieux, démuni et désabusé, il fait sienne l'idée que 'la Fortune est une putain \& vray vesse, qui s'abandonne à tout le monde, quelques foiz aux valletz mieux qu'aux Gentilz-hommes". Cette conception de la Fortune est, à l'évidence, celle que Calvin qualifie de 'resverie [qui] doit estre loin d'un cœur Chrestien' (I, xvi, 9, p. 234).

En ce qui concerne la littérature, certes, il faut faire la part des lieux communs. Les allusions à la Fortune font tellement partie du langage littéraire que souvent, même sans être de simples clichés, elles ne signifient pas grand-chose quant à la vérité religieuse. Prenons l'exemple des vers de Marot, cités plus haut: évoquer en passant le lieu commun de l'instabilité de la Fortune, ce n'est guère se prononcer sur la nature des choses. Un texte connu de Montaigne se rapporte à ces habitudes littéraires:

[B] le dire humain a ses formes plus basses et ne se doibt servir de la dignité, majesté, régence du parler divin. Je luy laisse, pour moy, dire, [C] 'verbis indisciplinatis' [B] fortune, d'estinée, accident, heur et malheur, et les Dieux et autres frases, selon sa mode ${ }^{6}$.

Selon lui la vérité religieuse doit être considérée ailleurs et dans un langage différent. Mais la distinction radicale entre discours humain et discours 'divin' n'élimine pas toutes les difficultés. Elle est peu convaincante surtout quand il s'agit d'un cri de colère aussi direct et intense que celui de Brantôme.

Chez Ronsard, la Fortune est présente à tel point qu'on l'a dit 'le grand poète

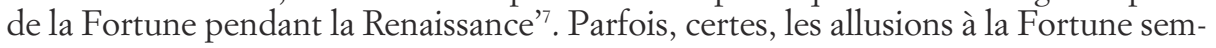

(4) Sur les tentatives médiévales de concilier christianisme et Fortune, voir H.R. PATCH, The Goddess Fortuna in Medieval Literature [1927], Londres, Frank Cass, 1967, ch. 1, surtout pp. 15-17 pour les Pères de l'Église; T. HunT, The Christianization of Fortune, Nottingham French Studies 38, (1999), pp. 95-113. Voir aussi, pour une analyse divergente, J.-C. MüHLETHALER, 'Quand Fortune, ce sont les bommes. Aspects de la démythification de la déesse, d'Adam de la Halle à Alain Chartier', La Fortune. Thèmes, représentations, discours, éd. Y. Foehr-Janssens et E. Métry, Genève, Droz, 2003, pp. 177-206.

(5) Discours sur les Colonels de l'Infanterie de France, éd. É. Vaucheret, Paris \& Montréal, Vrin Cosmos, 1973, p. 112.
(6) Essais, I, 56, éd. P. Villey \& V.-L. Saulnier, Paris, Presses Universitaires de France, 1965, p. 323. Voir Y. Bellenger, pp. 494-95.

(7) Y. Giraud, Ronsard et la fortune, La littérature de la Renaissance, éd. M. Soulié, Genève, Slatkine, 1984, pp.133-52 (p. 133). Voir aussi M. Quainton, Ronsard's Ordered Chaos, Manchester, Manchester University Press, 1980, surtout ch. 3; M. Dassonville, Ronsard. Étude bistorique et littéraire. IV. Grandeur et servitude, Genève, Droz, 1985, pp. 59-63; I. Silver, 'TycheFortune in the Epinician Odes of Pindar and in the Poetry of Ronsard', Il Tema della Fortuna..., pp. 151-60. 
blent de pure routine. Dans l'exemple suivant, son hostilité n'est mentionnée que pour rappeler discrètement et excuser poliment les défaites militaires de Henri II:

Et si Fortune, averse aux braves entreprises

De vostre majesté, ne les a toutes mises

A bienheueuse fin, toutesfois on a veu

Que vous avez osé \& que vous avez peu

(«La Paix, au Roy» [1559], v. 13-16; OC, IX, p. 104).

Le plus souvent, pourtant, la Fortune ronsardienne n'a rien d'un poncif et se charge de signification; nous le verrons de reste à propos de Mary Stuart.

Çà et là, la Fortune de Ronsard personnifie tout simplement le hasard. Le poète supplie ainsi Henri II de suivre une politique de paix:

...malheureux qui desire

Ainsi comme à trois detz hazarder son empire

Soubz le jeu de Fortune, \& duquel on ne sçait

Si l'incertaine fin doibt respondre au souhait

(«La Paix, au Roy» [1559], v. 205-08; OC, IX, p. 113).

Mais des cas aussi nets sont rares, et il est beaucoup plus fréquent que la Fortune apparaisse, non en abstraction personnifiée, mais en personnage doté de volonté personnelle. Ceux qui ont étudié récemment ce thème constatent que la Fortune ronsardienne est capricieuse et capable d'intervenir brutalement dans les affaires humaines. La question de ses rapports avec la Providence se pose donc de façon incontournable. Au terme d'une enquête fouillée, l'un des spécialistes y répond ainsi:

Although [Ronsard emphasises]... her omnipotence, inconstancy and hostility, nevertheless Fortune is equated with the prudence and wisdom of Divine Providence and, as Nemesis-Fortune, is the instrument through which a moral order of the universe is administered".

La Fortune aurait donc, malgré l'anarchie apparente de ses actes, un rôle moral à jouer dans un univers soumis à un ordre providentiel. Cette interprétation semble refléter en gros celle de la plupart des spécialistes ${ }^{10}$. Or, malgré cet accord général, j'espère montrer ici que deux poèmes, auxquels les chercheurs ne se sont intéressés qu'en passant ${ }^{11}$, évoquent une Fortune trop anarchique pour être conciliable avec un ordre providentiel. Il s'agit de l' «Elegie sur le depart de la Royne d'Escosse» [1561] (OC, XII, pp. 193-99) et, accessoirement, de l'«Elegie à la Royne d'Escosse» [1564] (ibid., pp. 277-84).

L'«Elegie sur le depart...» est inspirée par le retour en Écosse de Mary Stuart, veuve dès décembre 1560 du jeune François II. Pour en faciliter l'examen, on peut découper ainsi le poème:

I. (v. 1-10) Dénuement de la France sans Mary.

II. (v. 11-58) Biographie sommaire de Mary.

(8) Je renvoie aux Oeuvres complètes, éd P. Laumonier et autres, 20 tomes, Paris, Société des Textes Français Modernes, 1914-75, t. IX.

(9) QuAinTON, p. 221.

(10) Cf. Dassonville, p. 61; Silver, p. 159; GIRAUD, p. 145. (Mais l'étude de M. GIRAUD souligne la difficulté de cerner la pensée de Ronsard, qui nous propose nombre de conceptions différentes de la Fortune.)

(11) Par exemple, Quainton, p. 83; Silver, pp. 159-60. 
III. (v. 59-70) Invective contre la Fortune.

IV. (v. 71-76) Encore le dénuement de la France.

V. (v. 77-92) Obstacles imaginés par le poète pour empêcher le départ de la reine.

VI. (v. 93-114) Le départ de la reine et l'inspiration du poète.

Le noyau du poème est la biographie (II) et l'apostrophe à la Fortune (III), lesquelles renferment l'essentiel des maux imputés à la déesse. Le poète entrelace à ce thème des images de désordre; celles-ci, d'apparence anodine au début (I), en viennent à traduire sinistrement la puissance déchaînée de la Fortune (III, IV et V). Enfin, le long finale, qui nous intéresse moins ici, exprime la tristesse qui envahit à la fois le poète et sa poésie.

La partie II évoque quatre chapitres de la vie de Mary: son départ d'Écosse à l'âge de 5 ans (v. 16-24); son mariage et son avènement au trône de France (v. 25-30); la mort du roi, son mari (v. 31-52); la guerre civile en Écosse (v. 53-58). Le texte présente le tout comme l'œuvre de la Fortune, et tait ce qui ressortit à la volonté de Mary ou des siens. A cet égard, les vers suivants, sur le mariage de Mary et du dauphin François en 1558, sont typiques:

Et d'orpheline ensemble \& estrangere
(Hà, que tu es inconstante \& legere!)
La marias au fils de nostre Roy,
Qui depuis tint la France desoubs soy
Puis en l'ayant, ô Fortune insensée!
Jusqu'au sommet des grands honneurs poussée,
Tu as occis à seize ans son mary (v. 27-33).

Le vers 27 est peu exact: la mère de Mary vivait encore lors du mariage; Mary était à moitié française et surtout elle était appuyée en France par la puissante famille de Guise; elle n'était donc nullement la faible enfant que ce vers laisse entrevoir. Quant au mariage, en dépit des vers 27-29, il devait bien moins à la Fortune qu'au traité du 7 juillet 1548 qui l'avait prévu ${ }^{12}$.

La partie II, qui insiste ainsi sur la puissance de la Fortune, résume aussi son caractère. Dans le passage ci-dessus, où la Fortune améliore soudain la situation de Mary pour la replonger d'autant plus brutalement dans le malheur, son inconstance va jusqu'au délire (v. 28, 31), sa cruauté jusqu'au meurtre (v. 33). Ailleurs (v. 53-58), le texte reproche à la Fortune d'avoir fomenté 'traitrement' (v. 55) des guerres de religion au royaume écossais de Mary. La trahison - si trahison il y a - est sans doute celle des sujets rebelles; en accuser la Fortune, c'est une façon commode de résumer ses diverses perfidies envers la reine.

La partie biographique de l'élégie nous montre donc l'existence de Mary manipulée par une Fortune cruelle, folle, meurtrière et perfide. L'invective (III, v. 59-70) qui vise l'ensemble de ces torts, reproche à la Fortune son injustice morale:

Si la vertu, la bonté, la pitié,

La douceur jointe avec la gravité,

Les sainctes meurs, la chasteté de vie

N'ont resisté à ta cruelle envie,

Qu'esperons nous de nostre humanité? (v. 61-65).

(12) R. K. Marshall, Mary of Guise, Londres, Collins, 1977, pp. 165-66, 174. Ronsard n'ignorait pas l'aspect dynastique de ce mariage: son «Chant de liesse au Roy» [1559] félicite Henri II de voir son fils déjà roi d'Écosse et pouvant devenir roi d'Angleterre, grâce aux droits de Mary Stuart (v. 113-20, OC, IX, p. 138). 
On ne peut pas trop insister sur l'importance de cette accusation: elle exclut de considérer ici la fortune comme un agent de la justice divine et les maux qu'elle inflige comme des châtiments. Le vers 65 est une parenthèse: la Fortune est injuste et cruelle, les hommes le sont aussi. Cette réflexion, surgie dans l'attaque contre la Fortune, suggère avec un laconisme saisissant la généralité du mal sur la terre. Les vers suivants élargissent le champ de ces observations:

Le ciel là haut, ny sa divinité,

N'est pas bien seur, ny toutes ses Deesses,

Puis qu'icy bas nos divines Princesses,

Qui te devroient aux larmes inviter,

Contre le mal ne peuvent resister (v. 66-70).

Dans un contexte différent, on aurait pu croire que les allusions au ciel et aux déesses n'étaient amenées que par le qualificatif de 'divines' (v. 68) et ne servaient donc qu'à louer la beauté 'céleste' de la reine. Mais, ici, ces vers indiquent plutôt combien l'empire de la Fortune est vaste. Le ciel (entendons: la Providence) est si loin d'être maître de la Fortune qu'il en est menacé lui-même (v. 66-67). L'univers entier est en butte aux coups d'une Fortune malfaisante et envahissante. Telle est la conclusion où aboutissent ces parties centrales du poème.

Le pouvoir d'évocation de l'élégie tient en partie aux 'faits' qui y sont présentés, les souffrances de Mary et sa persécution par la Fortune. Il tient aussi, comme nous allons le voir, aux images. Le début (I) annonce le dénuement où tombera la France au départ de la reine. Une longue comparaison rapproche, d'une part, la France ainsi délaissée et, d'autre part, une série de choses plus ou moins viciées:

Comme un beau pré depoüillé de ses fleurs...

Ainsi perdra la France soucieuse

Ses ornemens, en perdant la beauté

Qui fut sa fleur, sa couleur, sa clarté (v. 1, 8-10).

Un 'beau pré' sans fleurs n'est sans doute pas beau, mais il s'agit du moins d'un défaut concevable. A la limite, pourtant, les défauts imaginés sont impossibles:

Comme le ciel, s'il perdoit ses estoilles,

La mer ses eaux... (v. 3-4).

Or l'évocation de phénomènes impossibles est un topos de la poésie amoureuse. Ronsard l'utilise, par exemple, pour proclamer sa fidélité à une maîtresse brune:

Plus tost les cieulx des mers seront couverts

Plus tost sans forme ira confus le monde:

Que je sois serf d'une maistresse blonde...

(Amours (1552), sonnet xxvi, v. 5-7; OC, IV, p. 29) ${ }^{13}$.

L'Elegie commence donc à la fois par un éloge flatteur de la beauté de Mary et par des images de désordre. Au long du poème paraîtront d'autres images de désordre; celles-ci, plus nettement sinistres, rappelleront et assombriront par analogie les premières. Et si, la lecture du poème finie, nous relisons le début, nous verrons que ces

(13) Sur les impossibles, voir RonSARD, Les Amours, éd. H. \& C. Weber, Paris, Classiques Gar- 
images liminaires préfigurent le désordre moral et les injustices atterrantes causés par la Fortune.

A ce point de vue, deux passages méritent notre attention. Le premier concerne la mort de François II. Les vers 31-33 évoquent la montée de la reine et, implicitement, sa rechute, lorsque le roi est 'occis' par la Fortune:

Puis en l'ayant, ô Fortune insensée!

Jusqu'au sommet des grands honneurs poussée,

Tu as occis à seize ans son mary.

Ensuite, le poème compare le mourant à des fleurs qui dépérissent:

Ny plus ny moins qu'en un jardin fleury

Meurt un beau Lys, quand la pluye pesante

Aggrave en bas sa teste languissante,

Ou comme au soir la rose perd couleur,

Et meurt sechée, alors que la chaleur

Boit son humeur qui la tenoit en vie,

Et fueille à fueille à bas tombe fanie (v. 34-40).

Le mouvement de chute imaginaire continue au ralenti (v. 36, 40), et entraîne aussi les fleurs qui figurent le roi mourant. Or le lis et la rose ne meurent pas, ils sont tués, l'un par la pluie (v. 35), l'autre par la chaleur (v. 38-39), tout comme le roi a été 'occis' par la Fortune.

Que les fleurs soient victimes de conditions opposées - pluie ou sécheresse - c'est une façon de suggérer que la Fortune a piégé le couple royal sans lui laisser d'issue. Il apparaît enfin que la Fortune a condamné François bien délibérément: dire 'en un jardin I Meurt un beau Lys' (v. 34-35), c'est indiquer qu'une seule fleur périt au milieu de beaucoup d'autres qui vivent.

Ce passage émouvant renvoie à l'ouverture du poème. Le lis et la rose rappellent les fleurs des vers 1 et 10; l'expression 'perd couleur' (v. 37) fait penser aux vers 2 et 10. La chute des feuilles (v. 40) rappelle le vers 5; et le sujet même, la disparition d'un roi, met l'ensemble des vers 33-40 en rapport avec le vers 6:

Un bois [s'il perdait] sa fueille, un antre son efroy,

Un grand palais la pompe de son Roy (v. 5-6).

De tels échos, qui relient les fleurs et le roi mourants aux anomalies évoquées au début du poème, confirment que cette ouverture n'était pas seulement un compliment, mais qu'elle annonçait les ravages de la Fortune ${ }^{14}$. Et cette métamorphose d'un topos emphatique mais inoffensif est elle-même comme un reflet de la puissance subversive de la Fortune.

Le second passage capital est dans notre partie V (v. 77-92), où le poète appelle de ses vœux quelque obstacle qui puisse obliger la reine à demeurer en France. Par exemple:

Puisse la mer la terre devenir,

Puisse la nef comme un rocher tenir

Au bord de l'eau... (v. 77-79).

(14) Un processus d'association analogue joue au milieu du poème, entre les vers 71-76 et les vers 66-67. 
Le parallèle est visible avec le vers 4:

Comme le ciel, s'il perdoit ses estoilles,

La mer ses eaux, la navire ses voiles (v. 3-4).

Mais le plus singulier de ces vœux est adressé à l'Écosse même, que le poète envisage comme une île, et qu'il incite à s'éloigner:

Hà, je voudrois que tu peusses ramer

De sur les flots, legere et vagabonde,

Comme un plongeon va leger de sur l'onde,

Pour t'en fuir longue espace devant

Le tard vaisseau lequel t'iroit suyvant,

Sans descharger au bord de ton rivage

La belle royne à qui tu doibs hommage (v. 86-92).

Ce vœu est surtout un cri de désespoir. Mais il y a pis. L'image du 'tard vaisseau' qui suit, sans pouvoir la rejoindre, une île fuyante (v. 89-90) est profondément mélancolique et troublante. Ce qui prédomine n'est pas l'idée du retour (impossible) de la reine, mais une impression d'isolement et d'abandon: on dirait que la Fortune, en donnant Mary 'aux flots de la marine' (v. 76), l'a condamnée à une errance perpétuelle ${ }^{15}$.

Le parallèle entre ces vœux et le début du poème constitue un paradoxe: dès le début, les maux infligés par la Fortune se concrétisent dans des images d'anomalies contre nature; le poète, en quête de remèdes, finit par imaginer et par désirer des anomalies non moins monstrueuses. Ce paradoxe saisissant traduit à la fois le désespoir du poète et le triomphe d'une Fortune malfaisante. Les images contribuent donc beaucoup à la valeur poétique de l'élégie et à l'expression des sentiments intenses qui y respirent. Ajoutons enfin que, au besoin, cette même intensité interdirait de ne voir dans l'élégie que des clichés sans signification réelle.

L'«Elegie sur le depart...» nous montre une Fortune autonome, anarchique et envahissante. L'«Elegie à la Royne d'Escosse» [1564] va encore plus loin. Ce poème évoque à son tour la beauté, désormais perdue, de Mary et le silence où son absence aurait réduit les Muses françaises (v. 1-52), ainsi que le courage (v. 65-78) et l'éloignement de la reine qui n'est plus accessible que par la pensée (v. 79-132). Dans un passage capital le poète s'interroge sur les malheurs de la reine (v. 53-64):

Hà, que bien peu s'en faut que remply de fureur,

Voyant vostre destin, je ne tombe en l'erreur

De ceux qui ont pensé qu'au plaisir de Fortune

Ce monde fut conduit sans prevoyance aucune (v. 53-56).

Cette mise au point, qui nie la suprématie de la Fortune et affirme le rôle de la Providence, semble remettre la Fortune à sa place dans un univers ordonné16 ${ }^{16}$. Mais le poète

(15) Le texte exploite une idée qui ne semblait sans doute pas tout à fait fantaisiste à une époque où les îles passaient pour être instables. (Voir, par exemple, F. Lestringant, 'L'Insulaire de Rabelais, ou la fiction en archipel (pour une lecture topographique du Quart Livre)', Rabelais en son demimillénaire, éd. J. Céard et J.-C. Margolin, Genève,
Droz, 1988, pp. 249-74. Cette méfiance à l'égard des îles ne réduit nullement le pouvoir d'évocation de la scène créée ici.

(16) Voir, en ce sens, Laumonier, XII, p. 280, note; Quainton, p. 64; Giraud, p. 145; Silver, p. 159. 
a beau rallier cette position orthodoxe, il la subvertit par ses aveux: la biographie de la reine le trouble au point qu'il devient fou ('remply de fureur') et qu'il est tenté ('bien peu s'en faut') de croire à l'hégémonie de la Fortune. Le trouble du poète reflète l'excès des maux qui ont affligé Mary; la tentation de croire à la toute-puissance de la Fortune révèle, à l'instant même où le poète affirme le rôle de la Providence, un doute radical sur celle-ci. Qui pis est, il lance aussitôt ce réquisitoire:

Ciel ingrat \& cruel, je te pry responds moy,

Responds, je te supply, que te fit nostre Roy,

Auquel si jeune d'ans tu as tranché la vie?

Que t'a fait son espouse \& sa fidelle amie,

De luy faire laisser le Sceptre si soudain,

Et vefve l'envoyer en un païs loingtain,

En la fleur de son age, ayant esmeu contre elle

Et contre sa grandeur sa terre naturelle? (v. 57-64).

Ces vers rappellent trois des quatre épisodes que l'«Elegie sur le depart ...» attribue à la Fortune. (Seul le départ d'Écosse manque complètement.) Mais ici, puisque le texte vient de souligner le rôle de la Providence, c'est celle-ci, sous le nom de 'Ciel', et non plus la Fortune, qu'il taxe de cruauté et d'injustice.

Cette interrogation brûlante reste sans réponse immédiate mais, après un développement sur l'absence de Mary, le poème se termine ainsi:

Ainsi Dieu qui a soing de vostre royauté,

A fait, miracle grand, naistre vostre beauté

Sur le bord estranger, comme chose laissée

Non pour les yeux de l'homme, ainçois pour la pensée (v. 129-32).

La Providence veille donc sur Mary; et l'absence physique convient à une beauté qui, trop exquise pour être appréciée par les sens, ne doit être contemplée que par l'esprit. Conclusion rassurante? Certes, elle propose une explication symbolique du retour de Mary en son Écosse natale. Mais elle laisse en suspens les questions plus épineuses comme celle de la mort de François II. En outre, ces vers rendent Dieu responsable des événements; le poète nous renvoie ainsi aux vers 57-64, qui réclament au ciel une explication des malheurs de la reine. D'un passage à l'autre, le ton s'est calmé, mais le fond du problème demeure: les maux subis par Mary sont cruellement injustes; et si cette injustice n'est pas imputable à la Fortune, à quoi l'attribuer sinon à la Providence? Tout en le voilant d'une conclusion à l'air consolateur, Ronsard repose le problème.

Avant de conclure, avouons que Ronsard ne traite pas toujours ainsi le sort de la reine: l'«Elegie à H. L'Huillier» (OC, XII, pp. 189-93), consacrée également au départ de Mary, est assez sereine. Il faut reconnaitre aussi que, dans l'ensemble de son œuvre, nos deux élégies sont trop exceptionnelles pour que l'on prête à Ronsard un scepticisme systématique. Mais l'élégie de 1564, comme l'«Elegie sur le depart...», montre que, face à l'existence tourmentée de la jeune veuve, Ronsard a peine à ne voir dans la Fortune qu'un agent de la justice divine; et elle montre aussi, plus clairement que l' «Elegie sur le depart...», qu'il peut en venir à remettre en question, - ne seraitce que fugitivement - la Providence même. 